\title{
Análisis de la rentabilidad de las universidades chilenas mediante la aplicación del sistema DuPont
}

\author{
Analysis of the profitability of chilean universities \\ through the application of the DuPont system
}

\author{
Alejandro Diaz Ramos ${ }^{1}$
}

\begin{abstract}
RESUMEN
Mediante el presente artículo se realizó un análisis de los niveles de rentabilidad generados por las universidades chilenas. El estudio es de carácter descriptivo y se realizó sobre las cifras reportadas en los estados financieros anuales que las entidades publican a través del Sistema de Información de la Educación Superior (SIES) del Ministerio de Educación. El análisis se realiza mediante la aplicación del modelo de DuPont conocido como el sistema que permite la desagregación de las métricas de rentabilidad en indicadores de eficiencia interna que representan determinadas áreas de la administración de las entidades. El trabajo permitió evaluar la evolución de los indicadores de rentabilidad de las entidades, junto con determinar las diferencias de desempeño dependiendo del grupo de universidades clasificadas según su naturaleza jurídica y financiamiento.
\end{abstract}

Palabras Claves: análisis financiero, estados financieros, modelo de Dupont.

Recepción: 31/08/2020. Aprobación: 03/12/2020.

\begin{abstract}
Through this article, an analysis of the profitability levels generated by the chilean universities. The analysis is descriptive in nature and is carried out on the figures reported in the annual financial statements that each institution publishes through the Higher Education Information System (HEIS) of the Ministry of Education. The analysis is carried out by applying the DuPont Model known as the system that allows the disaggregation of profitability metrics into internal efficiency indicators that represent certain areas of the entities management. The work allowed evaluating the evolution of the profitability indicators of Chilean universities, along with determining performance differences depending on the university group classified according to its legal nature and financing.
\end{abstract}

Keywords: financial analysis, financial statements, DuPont model.

1 Universidad de Atacama, Facultad de Ingeniería, Copiapó, Chile, alejandro.diaz@uda.cl 


\section{INTRODUCCIÓN}

El análisis financiero y en específico las evaluaciones de la rentabilidad generada por las organizaciones constituyen herramientas que se han aplicado durante décadas para evaluar la capacidad que tienen las entidades para generar márgenes o excedentes, ya sea a través de la medición del retorno financiero que entrega la inversión en activos productivos (ROA) o bien midiendo el retorno sobre el capital invertido (ROE).

En el sistema de educación superior chileno la medición y análisis de la rentabilidad de las universidades tiene aplicación desde el año 2006, cuando mediante la Ley 20.044 (publicada en el Diario Oficial el 23 de agosto de 2005) del Ministerio de Educación se comienza a exigir a las universidades estatales la publicación de sus estados financieros auditados. En el mismo año 2006 se promulga en el Diario Oficial el 23 de octubre la Ley 20.129 del mismo Ministerio, norma mediante la cual se establece el Sistema Nacional de Aseguramiento de la Calidad de la Educación Superior, sistema que dentro de sus dimensiones y criterios de evaluación establece aquella que dice relación con la evaluación de la sostentabilidad económica y financiera de las entidades de educación superior.

De acuerdo a OCDE $(2004)^{2}$ se establecen las directrices a seguir por las instituciones de educación superior (IES) con el propósito de asegurar la sustentabilidad económica. Dentro de estas reglas se destaca las condiciones relacionadas con la fijación de precios, la recuperación de costos y generación de ingresos. De acuerdo a los criterios fijados por la OCDE en relación a la sustentabilidad financiera de las IES, se encuentra la realización de operaciones sostenibles por tanto estas entidades deberán generar ingresos a partir de sus actividades ordinarias que sean suficientes para cubrir sus costos normales de operación.

Asimismo, CNA-Chile (2018) ${ }^{3}$ establece que la evaluación de la sustentabilidad financiera se encuentra inserta dentro de una de las áreas de acreditación obligatoria para las IES: La gestión institucional. Dentro de los procesos de acreditación de la calidad aplicados a las IES, surge como tema específico el comprobar si las entidades evaluadas disponen de los recursos financieros para materializar y sustentar en el mediano plazo el proyecto institucional declarado. CNAChile ha determinado que dicha evaluación se efectúe mediante la contratación de un consultor externo e independiente, que debe pronunciarse respecto de la sustentabilidad financiera de las entidades examinadas. Fruto de esta evaluación se emite un Informe de Sustentabilidad Financiera (ISF), documento que la CNA pone a disposición de cada comité de pares evaluadores y de las autoridades de la institución estudiada. Este análisis se refiere exclusivamente al comportamiento financiero de cada institución y la factibilidad de que disponga de los recursos necesarios para desarrollar su proyecto educativo en un horizonte de 5 años. Con lo anterior queda de manifiesto la importancia que reviste para la gestión universitaria, la monitorización permanente de aquellos indicadores financieros que den cuenta de la capacidad que tienen las IES para generar márgenes o excedentes que den señales de sustentabilidad económica y financiera de las entidades. En esta línea, el método Dupont permite examinar de manera simultánea indicadores de eficiencia y rentabilidad, además de la interacción entre ambos tipos de ratios para la

2 Organización para la Cooperación y el Desarrollo Económico.

3 Comisión Nacional de Acreditación de Chile. 
determinación de los ratios ROA y ROE según Dehning \& Stratopoulos (2002). Con tal objetivo se relaciona la información del estado de resultados cuantificando la capacidad de las empresas para convertir ventas en utilidades, y por otra parte se relaciona la información del estado de situación financiera midiendo la capacidad que tienen las empresas para generar ingresos a partir de los activos en los que ha invertido. Son cinco indicadores en que se desagrega el ROE según el modelo de Dupont, permitiendo cuantificar el crecimiento de una firma considerando que las utilidades y por tanto la rentabilidad se generan a partir de cuatro dimensiones: Margen sobre las ventas, rotación de activos, apalancamiento financiero, carga por intereses y carga tributaria de acuerdo a Berstein L \& Wild J. (1998); Stickney C.\& Brown P. (1999); Revsine L., Collins D. \& Johnson W. (2005). El modelo de Dupont, creado en la década de 1920 por el ingeniero Donaldson Brown mientras se desempeñaba como director financiero y ejecutivo de las Corporaciones Dupont y General Motors, es uno de los métodos de análisis financiero de mayor uso según Gutiérrez Hidalgo F. (2005). De acuerdo a Gitman L. (2003), El análisis de Dupont constituye una técnica de investigación que tiene por objetivo hallar las funciones administrativas responsables del desempeño financiero de la entidad, tomando en consideración todos los elementos de las actividades financieras del negocio. El modelo Dupont se basa en la interrelación de determinados indicadores financieros que conjuntamente explican la capacidad de la entidad para generar ganancias y rentabilidad.

La ecuación muestra la desagregación del ROE según lo plantea el modelo de Dupont.

$$
R O E(\%)=\frac{\text { Utilidad neta }}{E B T} \times \frac{E B T}{E B I T} \times \frac{E B I T}{\text { Ventas }} \times \frac{\text { Ventas }}{\text { Activo Total }} \times \frac{\text { Activo Total }}{\text { Patrimonio }}
$$

A continuación, se describen cada uno de los indicadores expresados en la ecuación anterior.

Carga por impuestos: Se calcula a partir de la relación entre utilidad neta/EBT, donde $\mathrm{EBT}^{4} \mathrm{co}-$ rresponde a la utilidad antes de impuestos. Conforme la entidad tenga una mayor carga tributaria el indicador tenderá a cero dado que la utilidad neta se reduce producto del pago de impuestos a las ganancias reduciendo así el ROE. En caso que la entidad tenga una baja carga tributaria el indicador tenderá a uno, dado que a menor carga de impuestos la utilidad neta será similar al EBT aumentando el ROE.

Carga por intereses: Se calcula a partir de la relación EBT/EBIT ${ }^{5}$. EBIT corresponde a las utilidades antes de intereses e impuestos o resultado operacional. Conforme la entidad tenga mayor carga por gastos financieros tendrá un menor EBT por tanto el indicador tenderá a cero reduciendo el ROE. Por el contrario, en caso de que la entidad tenga baja carga por gastos financieros el EBT convergerá con el EBIT, por tanto, el indicador tenderá a uno aumentando así el ROE. Este indicador eventualmente podría ser mayor a uno cuando el EBT sea mayor que el EBIT, lo que sucedería cuando la entidad junto con tener bajos gastos financieros además registra ganancias no operacionales ${ }^{6}$.

4 Earnings Before Taxes por sus siglas en inglés.

5 Earnings Before Interest and Taxes por sus siglas en inglés.

6 Las ganancias no operacionales podrían explicarse por ingresos financieros, reconocimiento de ganancias en entidades relacionadas, ganancias por diferencias de cambio y/o unidades de reajuste. 
Rentabilidad operacional: Indicador calculado a partir de la relación entre el EBIT/Ventas. Expresa el margen operacional en relación a los ingresos por ventas de la entidad.

Rotación de activos: Ratio de eficiencia que se calcula a partir de la relación entre los ingresos por ventas y los activos totales de la entidad. El indicador evidenciará mayores niveles de eficiencia en la medida que la entidad genere mayores ventas a partir de los activos de los que dispone.

Solvencia patrimonial: Indicador de apalancamiento que se calcula a partir de la relación entre los activos totales y el patrimonio de la entidad. A menor nivel de endeudamiento para financiar inversiones en activos el indicador tenderá a uno. Cuando la entidad comienza a contratar deuda el indicador será mayor a uno, por lo que aumentará el ROE. El mayor apalancamiento financiero de la entidad le permite aumentar los niveles de rentabilidad.

Entendiendo la importancia que reviste el análisis de la rentabilidad de las IES en Chile, además de la utilidad que ofrece el modelo de Dupont para realizar dicho análisis, las preguntas de investigación que motivan este trabajo son: ¿Qué niveles de rentabilidad generan las universidades y cuál ha sido su evolución en el tiempo?, ¿Existen diferencias en la estructura de rentabilidad dependiendo de la naturaleza de la institución, pudiendo ser estatal, privada con financiamiento estatal o bien totalmente privada?, y ¿Cómo la rentabilidad de las ventas, el apalancamiento financiero, los costos financieros y la carga tributaria determinan la rentabilidad sobre el patrimonio de las universidades?

\section{METODOLOGÍA, MATERIALES Y MÉTODOS}

Para el cumplimiento de los propósitos del presente trabajo, se utilizaron datos e información reportados en los estados financieros anuales auditados de las universidades chilenas entre los años 2014 al 2018. Al año 2018 fueron 57 universidades del país que entregaron sus informes financieros a través del Sistema de Información para la Educación Superior del Ministerio de Educación (SIES). Del total de estas instituciones 18 (31\%) corresponden a universidades estatales, $9(16 \%)$ a universidades privadas con financiamiento estatal ${ }^{7}$ y $30(53 \%)$ a universidades privadas. En el periodo la muestra fue acotada a 54 instituciones lo que implicó el análisis de 270 informes financieros anuales auditados. Se excluyeron de la muestra seis universidades privadas que en el periodo no registran información en algunos años y además se encuentran en situación de quiebra o cierre. También se excluyeron de la muestra dos universidades estatales creadas en el año 2015 como son los casos de las Universidades de O’Higgins y Aysén. Con la información financiera pertinente sistematizada en una base de datos, se construyó el conjunto de indicadores propios del sistema Dupont y sobre éstos se realizó un análisis evolutivo de cinco años, sustentado fundamentalmente en la caracterización del conjunto de indicadores a partir de estadísticos descriptivos.

7 Estas nueve universidades privadas que forman parte del Consejo de Rectores de las Universidades Chilenas (CRUCH) coloquialmente se denominan universidades del G9. 


\section{RESULTADOS}

Una vez construidos los indicadores del sistema Dupont (rentabilidad operacional, eficiencia en la rotación de activos, endeudamiento, carga financiera y carga tributaria), sobre dichas métricas se calcularon estadísticos descriptivos como media, desviación estándar, mínimos y máximos. Estos estadísticos han permitido caracterizar la evolución de las relaciones determinantes del ROE de las universidades chilenas según se muestra a continuación en la tabla 1.

\section{Tabla 1}

Evolución y descomposición del ROE según el método de Dupont para los años 2014 al 2018.

\begin{tabular}{|c|c|c|c|c|c|c|}
\hline \multirow[b]{2}{*}{ Año } & \multirow[b]{2}{*}{ Componente } & \multirow[b]{2}{*}{ Indicador } & \multicolumn{4}{|c|}{ Sistema Universidades $(n=54)$} \\
\hline & & & media & $\begin{array}{c}\text { desv. } \\
\text { estándar }\end{array}$ & minimo & máximo \\
\hline \multirow{6}{*}{2018} & Carga por Impuestos & Utilidad Neta/EBT & 1,00 & 0,02 & 0,89 & 1,05 \\
\hline & Carga por Intereses & EBT/EBIT & 0,39 & 5,46 & 38,12 & 6,91 \\
\hline & Operación & EBIT/Ventas & $3,46 \%$ & $9,51 \%$ & $-45,70 \%$ & $24,80 \%$ \\
\hline & Eficiencia & Ventas/activo total & 0,66 & 0,39 & 0,30 & 2,70 \\
\hline & Deuda/ Solvencia & Activo Total/Patrim. & 2,28 & 2,56 & 1,10 & 15,21 \\
\hline & \multicolumn{2}{|c|}{ ROE } & $-6,57 \%$ & $70,33 \%$ & $-497,60 \%$ & $28,89 \%$ \\
\hline \multirow{6}{*}{2017} & Carga por Impuestos & Utilidad Neta/EBT & 1,00 & 0,01 & 0,93 & 1,02 \\
\hline & Carga por Intereses & EBT/EBIT & 1,03 & 1,08 & 0,57 & 7,60 \\
\hline & Operación & EBIT/Ventas & $4,74 \%$ & $10,41 \%$ & $-35,35 \%$ & $50,21 \%$ \\
\hline & Eficiencia & Ventas/activo total & 0,67 & 0,42 & 0,27 & 2,96 \\
\hline & Deuda/ Solvencia & Activo Total/Patrim. & 2,92 & 4,85 & 1,10 & 28,74 \\
\hline & \multicolumn{2}{|c|}{ ROE } & $4,11 \%$ & $21,07 \%$ & $-97,33 \%$ & $62,78 \%$ \\
\hline \multirow{6}{*}{2016} & Carga por Impuestos & Utilidad Neta/EBT & 1,00 & 0,02 & 0,94 & 1,11 \\
\hline & Carga por Intereses & EBT/EBIT & 1,14 & 2,54 & $-\quad 3,37$ & 18,10 \\
\hline & Operación & EBIT/Ventas & $2,04 \%$ & $17,87 \%$ & $-108,69 \%$ & $36,32 \%$ \\
\hline & Eficiencia & Ventas/activo total & 0,65 & 0,34 & 0,25 & 1,73 \\
\hline & Deuda/ Solvencia & Activo Total/Patrim. & 2,09 & 3,93 & 14,50 & 23,46 \\
\hline & \multicolumn{2}{|c|}{$\begin{array}{l}\text { ROE } \\
\end{array}$} & $5,16 \%$ & $35,15 \%$ & $-144,70 \%$ & $137,04 \%$ \\
\hline \multirow{6}{*}{2015} & Carga por Impuestos & Utilidad Neta/EBT & 1,00 & 0,03 & 0,92 & 1,12 \\
\hline & Carga por Intereses & EBT/EBIT & 0,84 & 1,76 & 6,21 & 8,97 \\
\hline & Operación & EBIT/Ventas & $0,88 \%$ & $26,86 \%$ & $-179,90 \%$ & $34,61 \%$ \\
\hline & Eficiencia & Ventas/activo total & 0,64 & 0,33 & 0,25 & 1,60 \\
\hline & Deuda/ Solvencia & Activo Total/Patrim. & 2,30 & 3,76 & $\begin{array}{l}-\quad 5,04 \\
\end{array}$ & 27,17 \\
\hline & \multicolumn{2}{|c|}{ ROE } & $3,11 \%$ & $31,62 \%$ & $-134,53 \%$ & $123,72 \%$ \\
\hline \multirow{6}{*}{2014} & Carga por Impuestos & Utilidad Neta/EBT & 0,98 & 0,12 & 0,17 & 1,15 \\
\hline & Carga por Intereses & EBT/EBIT & 12,28 & 93,89 & 689,41 & 2,00 \\
\hline & Operación & EBIT/Ventas & $4,13 \%$ & $12,08 \%$ & $-54,98 \%$ & $31,54 \%$ \\
\hline & Eficiencia & Ventas/activo total & 0,62 & 0,32 & 0,22 & 1,63 \\
\hline & Deuda/ Solvencia & Activo Total/Patrim. & 5,57 & 21,77 & 3,81 & 157,29 \\
\hline & \multicolumn{2}{|c|}{ ROE } & $47,50 \%$ & $270,29 \%$ & $-51,66 \%$ & $1945,27 \%$ \\
\hline
\end{tabular}

Fuente: Elaboración propia. 
A partir de los resultados obtenidos se observa un deterioro del ROE promedio de las instituciones universitarias. Para el año 2014 el ROE promedio del sistema universitario chileno llega a un $47,5 \%$ para pasar a un $-6,57 \%$ en 2018 . Es importante observar que el ROE promedio del sistema en el año 2014 se encuentra fuertemente influenciado por el resultado de dos universidades en particular, las cuales por tener un bajo patrimonio muestran un indicador de solvencia que introduce un importante efecto multiplicador al ROA para obtener un ROE observado de $1.945,27 \%$ y 442,33\% en los casos de las Universidades Bolivariana y La República respectivamente. Si se consideran ambos casos como outliers y se extraen de la muestra, el ROE promedio del sistema para el año 2014 arroja un 3,41\% y desviación estándar del indicador de 16,34\%. El ROE muestra una tendencia bajista en los cinco años analizados, al mismo tiempo que se evidencia un aumento en la dispersión del indicador considerando que la desviación estándar al año 2018 llega a un 70,33\%. Vale mencionar que en el año 2018 el ROE promedio del sistema se encuentra fuertemente influenciado por los resultados de la Universidad Gabriela Mistral (UGM), entidad que anota un ROE de $-497,6 \%$. Si consideramos dicho resultado como un valor fuera de rango y se extrae de la muestra, el ROE promedio del sistema para el año 2018 aumenta a 2,69\%, siendo todavía el menor ROE del periodo analizado.

Mediante la figura 1 se muestra la evolución de los indicadores ROE y rentabilidad operacional (EBIT/ Ventas) como promedio del sistema de universidades chilenas.

\section{Figura 1}

Evolución del ROE y rentabilidad operacional del sistema de universidades chilenas.

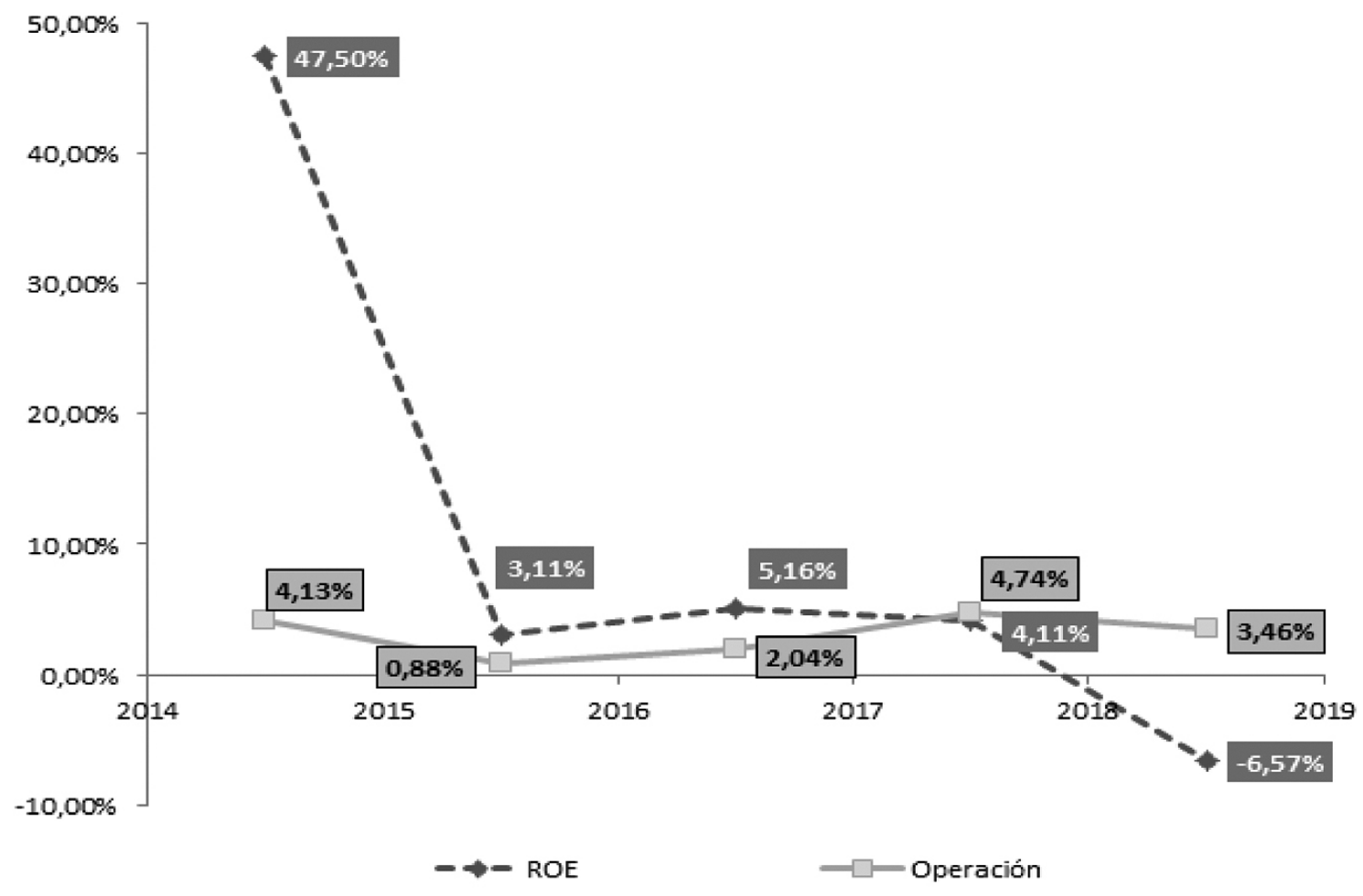

Fuente: Elaboración propia. 
En la figura anterior se puede apreciar una relativa estabilidad en la evolución de la rentabilidad operacional promedio del sistema considerando que al año 2014 el indicador arrojaba un resultado de 4,13\% mientras que al año 2018 llega a 3,46\%. La volatilidad del indicador de rentabilidad operacional desciende en el periodo, considerando una desviación estándar de $12,08 \%$ al año 2014 y de 9,51\% al año 2018. Por su parte, los indicadores de eficiencia, endeudamiento, carga financiera y carga tributaria, muestran la siguiente evolución según se muestra en la figura 2.

\section{Figura 2}

Evolución de los indicadores de eficiencia, endeudamiento, carga por intereses y carga tributaria del sistema de universidades chilenas.

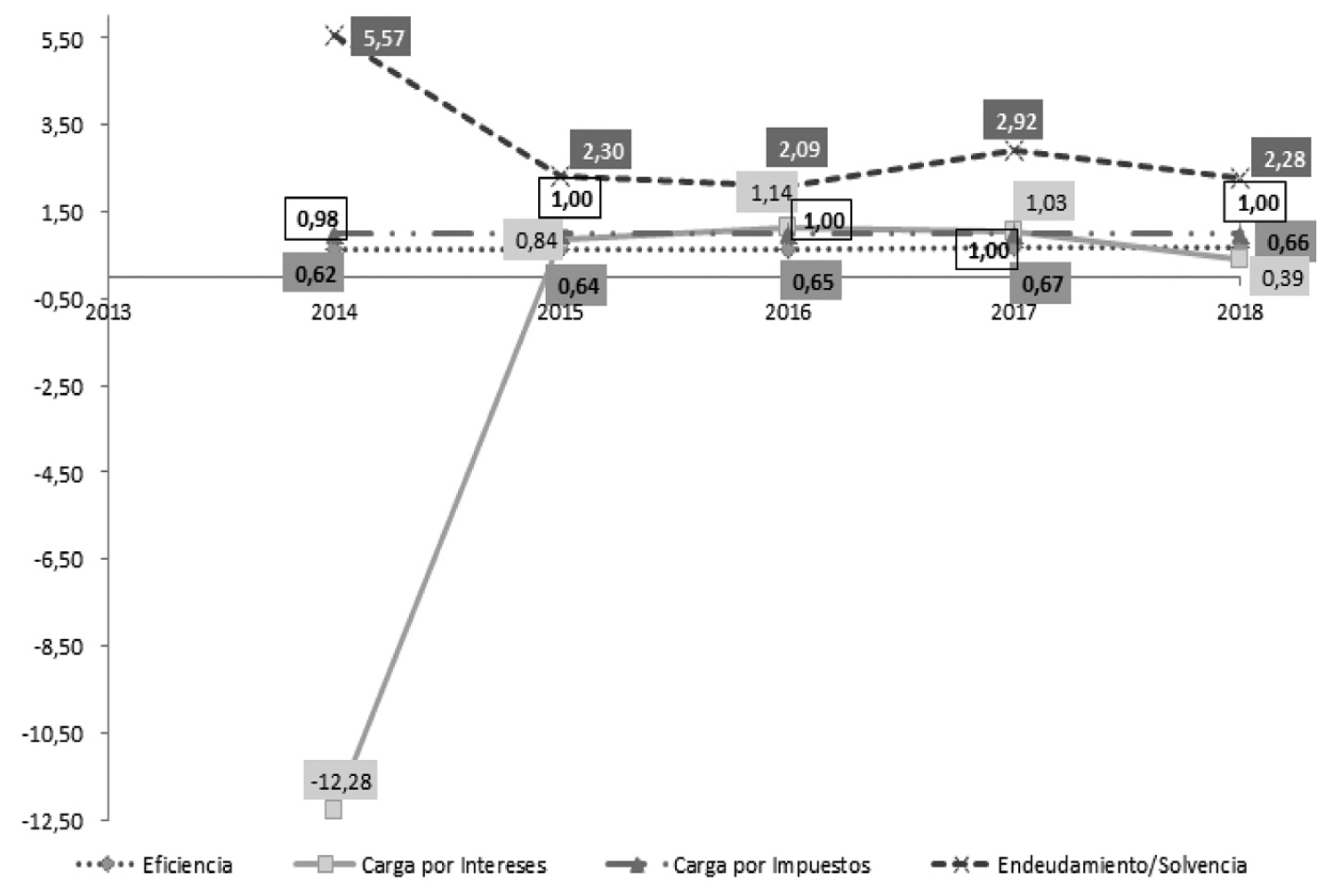

Fuente: Elaboración propia.

Para el indicador de eficiencia por la rotación de activos (figura 2) se observa una evolución estable con un resultado de 0,62 veces al año 2014 y de 0,66 veces al año 2018. Este coeficiente indica que en promedio las universidades generan ingresos operacionales anuales en un rango que va entre 0,62 y 0,66 veces en relación al total de activos que administran las instituciones. Por su parte la carga de intereses financieros evidencia un resultado negativo de $-12,28$ en el año 2014 cifra que se explica por un grupo de universidades que presenta resultados negativos 
tanto a nivel de EBIT como EBT. En el año 2014 se detecta una institución con una carga por intereses importantemente negativa siendo el caso de Universidad Internacional SEK, la cual muestra un indicador de $-689,41$ veces. Si se diera tratamiento de outliers a este resultado, extrayéndolo de la muestra, el ratio promedio de carga por intereses para el año 2014 llegaría a 0,5 veces. Para el periodo comprendido entre los años 2015 al 2018, la carga de intereses promedio de las universidades muestra una tendencia a la baja considerando que al año 2015 el ratio alcanzaba un valor de 0,84 veces llegando a 0,39 veces al año 2018. La tendencia del indicador refleja la divergencia de los resultados antes de impuestos frente a los resultados antes de intereses e impuestos, revelando un aumento en los costos financieros incurridos por las universidades lo cual deteriora el ROE del sistema. El indicador de solvencia evidencia estabilidad considerando que al año 2014 es de 5,57 veces el que se incrementa por los niveles de apalancamiento de dos universidades en particular (Bolivariana y La República). Al extraer los resultados de dichas instituciones como outliers, el indicador de solvencia promedio del sistema llegaría a 2,05 veces. Para el resto del periodo la estabilidad del indicador se refleja en un resultado de 2,3 veces y 2,28 veces para los años 2015 y 2018 respectivamente. El resultado indica que en promedio las universidades chilenas mantienen una estructura de financiamiento donde la inversión en activos se encuentra financiada en un $43 \%$ con patrimonio y un $57 \%$ con pasivos, nivel de apalancamiento que contribuye positivamente a la determinación del ROE. Finalmente, en relación al indicador que mide la carga por impuestos se observa que éste en promedio es virtualmente igual a 1 para todo el periodo. Lo anterior revela la nula carga tributaria de las universidades chilenas.

Con el propósito de profundizar el análisis de la descomposición del ROE según el carácter de las instituciones universitarias se distinguirá entre universidades: Estatales, privadas CRUCH y privadas-privadas. Mediante la tabla 2 se muestra la evolución de los indicadores determinantes del ROE para el periodo comprendido entre los años 2014 al 2018 según tipo de universidad. 
Tabla 2

Evolución y descomposición del ROE mediante el método de Dupont según tipo de universidad.

\begin{tabular}{|c|c|c|c|c|c|c|c|c|c|c|c|c|c|c|}
\hline \multirow{2}{*}{ Año } & \multirow{2}{*}{ Componente } & \multirow{2}{*}{ Indicador } & \multicolumn{4}{|c|}{ Universidades Estatales $(n=16)$} & \multicolumn{4}{|c|}{$\begin{array}{l}\text { Universidades Privadas CRUCH } \\
\qquad(\mathrm{n}=9)\end{array}$} & \multicolumn{4}{|c|}{ Universidades Privadas $(n=29)$} \\
\hline & & & media & $\begin{array}{c}\text { desv. } \\
\text { estándar }\end{array}$ & minimo & máximo & media & $\begin{array}{c}\text { desv. } \\
\text { estándar }\end{array}$ & minimo & máximo & media & $\begin{array}{l}\text { desv. } \\
\text { estándar }\end{array}$ & minimo & máximo \\
\hline \multirow{6}{*}{2018} & Carga por Impuestos & Utilidad Neta/EBT & 1,00 & 0,01 & 0,99 & 1,05 & 0,99 & 0,04 & 0,89 & 1,03 & 1,00 & 0,01 & 0,97 & 1,02 \\
\hline & Carga por Intereses & EBT/EBIT & 1,52 & 1,67 & 0,31 & 6,91 & 3,52 & $12,97-$ & 38,12 & 1,17 & 0,98 & 0,92 & 0,01 & 5,37 \\
\hline & Operación & EBIT/Ventas & $1,21 \%$ & $5,89 \%$ & $-15,15 \%$ & $8,03 \%$ & $7,05 \%$ & $5,61 \%$ & $0,04 \%$ & $17,73 \%$ & $3,59 \%$ & $11,69 \%$ & $-45,70 \%$ & $24,80 \%$ \\
\hline & Eficiencia & Ventas/activo total & 0,46 & 0,11 & 0,30 & 0,61 & 0,55 & 0,15 & 0,35 & 0,83 & 0,81 & 0,48 & 0,32 & 2,70 \\
\hline & Deuda/Solvencia & Activo Total/Patrim. & 1,37 & 0,21 & 1,10 & 1,75 & 1,64 & 0,41 & 1,20 & 2,46 & 2,99 & 3,34 & 1,19 & 15,21 \\
\hline & \multicolumn{2}{|c|}{ ROE } & $0,65 \%$ & $3,22 \%$ & $-5,70 \%$ & $5,17 \%$ & $5,86 \%$ & $5,89 \%$ & $-0,90 \%$ & $17,67 \%$ & $-14,42 \%$ & $95,93 \%$ & $-497,60 \%$ & $28,89 \%$ \\
\hline \multirow{6}{*}{2017} & Carga por Impuestos & Utilidad Neta/EBT & 1,00 & 0,01 & 0,98 & 1,00 & 0,99 & 0,03 & 0,93 & 1,02 & 1,00 & 0,01 & 0,96 & 1,01 \\
\hline & Carga por Intereses & EBT/EBIT & 1,63 & 1,77 & $-\quad 0,13$ & 7,60 & 0,92 & 0,38 & 0,44 & 1,75 & 0,74 & 0,44 & - $\quad 0,57$ & 1,83 \\
\hline & Operación & EBIT/Ventas & $1,77 \%$ & $3,76 \%$ & $-4,53 \%$ & $6,53 \%$ & $7,97 \%$ & $6,85 \%$ & $-1,30 \%$ & $17,54 \%$ & $5,37 \%$ & $13,24 \%$ & $-35,35 \%$ & $50,21 \%$ \\
\hline & Eficiencia & Ventas/activo total & 0,46 & 0,10 & 0,29 & 0,60 & 0,58 & 0,16 & 0,41 & 0,89 & 0,81 & 0,52 & 0,27 & 2,96 \\
\hline & Deuda/Solvencia & Activo Total/Patrim. & 1,39 & 0,26 & 1,10 & 1,98 & 1,66 & 0,42 & 1,21 & 2,42 & 4,16 & 6,41 & 1,19 & 28,74 \\
\hline & \multicolumn{2}{|c|}{ ROE } & $0,63 \%$ & $3,03 \%$ & $-6,78 \%$ & $4,54 \%$ & $7,57 \%$ & $7,99 \%$ & $-1,76 \%$ & $20,03 \%$ & $4,96 \%$ & $28,38 \%$ & $-97,33 \%$ & $62,78 \%$ \\
\hline \multirow{6}{*}{2016} & Carga por Impuestos & Utilidad Neta/EBT & 1,01 & 0,03 & 0,99 & 1,11 & 1,01 & 0,03 & 0,99 & 1,09 & 1,00 & 0,02 & 0,94 & 1,05 \\
\hline & Carga por Intereses & EBT/EBIT & 1,01 & 0,54 & 0,18 & 2,02 & 0,83 & 0,33 & 0,49 & 1,62 & 1,31 & 3,46 & 3,37 & 18,10 \\
\hline & Operación & EBIT/Ventas & $3,76 \%$ & $5,16 \%$ & $-10,14 \%$ & $12,85 \%$ & $7,34 \%$ & $4,88 \%$ & $2,62 \%$ & $16,24 \%$ & $-0,56 \%$ & $23,78 \%$ & $-108,69 \%$ & $36,32 \%$ \\
\hline & Eficiencia & Ventas/activo total & 0,46 & 0,10 & 0,29 & 0,71 & 0,57 & 0,18 & 0,36 & 0,91 & 0,78 & 0,41 & 0,25 & 1,73 \\
\hline & Deuda/Solvencia & Activo Total/Patrim & 1,34 & 0,21 & 1,11 & 1,79 & 1,71 & 0,49 & 1,20 & 2,60 & 2,62 & 5,34 & 14,50 & 23,46 \\
\hline & \multicolumn{2}{|c|}{ ROE } & $1,70 \%$ & $4,07 \%$ & $-11,54 \%$ & $6,49 \%$ & $5,78 \%$ & $4,17 \%$ & $1,21 \%$ & $13,75 \%$ & $6,87 \%$ & $48,11 \%$ & $-144,70 \%$ & $137,04 \%$ \\
\hline \multirow{6}{*}{2015} & Carga por Impuestos & Utilidad Neta/EBT & 0,99 & 0,03 & 0,92 & 1,03 & 1,01 & 0,03 & 0,99 & 1,10 & 1,00 & 0,03 & 0,94 & 1,12 \\
\hline & Carga por Intereses & EBT/EBIT & 1,24 & 2,50 & 2,27 & 8,97 & 0,83 & 0,62 & 0,13 & 2,27 & 0,63 & 1,50 & 6,21 & 3,00 \\
\hline & Operación & EBIT/Nentas & $4,75 \%$ & $5,34 \%$ & $0,09 \%$ & $18,75 \%$ & $5,79 \%$ & $4,92 \%$ & $0,10 \%$ & $17,09 \%$ & $-2,77 \%$ & $36,24 \%$ & $-179,90 \%$ & $34,61 \%$ \\
\hline & Eficiencia & Ventas/activo total & 0,45 & 0,10 & 0,25 & 0,65 & 0,58 & 0,19 & 0,41 & 0,97 & 0,76 & 0,38 & 0,27 & 1,60 \\
\hline & Deuda/ Solvencia & Activo Tota1/Patrim. & 1,31 & 0,20 & 1,11 & 1,73 & 1,73 & 0,57 & 1,18 & 2,87 & 3,03 & 5,04 & 5,04 & 27,17 \\
\hline & \multicolumn{2}{|c|}{ ROE } & $1,88 \%$ & $2,73 \%$ & $-1,69 \%$ & $8,96 \%$ & $3,28 \%$ & $2,59 \%$ & $0,12 \%$ & $8,45 \%$ & $3,73 \%$ & $43,43 \%$ & $-134,53 \%$ & $123,72 \%$ \\
\hline \multirow{6}{*}{2014} & Carga por Impuestos & Utilidad Neta/EBT & 0,99 & 0,01 & 0,96 & 1,00 & 1,01 & 0,07 & 0,91 & 1,15 & 0,96 & 0,16 & 0,17 & 1,03 \\
\hline & Carga por Intereses & EBT/EBIT & 0,99 & 0,59 & 0,04 & 2,00 & 0,55 & $0,53-$ & 0,52 & 1,17 & 23,58 & 128,07 & 689,41 & 1,45 \\
\hline & Operación & EBIT/Ventas & $5,91 \%$ & $5,50 \%$ & $-3,67 \%$ & $17,39 \%$ & $6,07 \%$ & $4,39 \%$ & $-0,41 \%$ & $11,69 \%$ & $2,54 \%$ & $15,78 \%$ & $-54,98 \%$ & $31,54 \%$ \\
\hline & Eficiencia & Ventas/activo total & 0,45 & 0,11 & 0,22 & 0,63 & 0,56 & 0,19 & 0,40 & 0,95 & 0,74 & 0,39 & 0,25 & 1,63 \\
\hline & Deuda/Solvencia & Activo Total/Patrim. & 1,28 & 0,17 & 1,11 & 1,68 & 1,71 & 0,59 & 1,17 & 2,94 & 9,14 & 29,47 & 3,81 & 157,29 \\
\hline & \multicolumn{2}{|c|}{ ROE } & $3,30 \%$ & $4,14 \%$ & $-3,66 \%$ & $12,15 \%$ & $3,22 \%$ & $2,12 \%$ & $0,10 \%$ & $6,64 \%$ & $85,63 \%$ & $367,46 \%$ & $-51,66 \%$ & $1945,27 \%$ \\
\hline
\end{tabular}

Fuente: Elaboración propia.

De acuerdo a los resultados el grupo de universidades que evidencia mejor ROE promedio y al mismo tiempo la menor dispersión corresponde a las universidades privadas CRUCH con rentabilidad promedio que va desde un 3,22\% en el año 2014 y 5,86\% en 2018. Si bien el grupo de las entidades privadas-privadas muestra el mejor resultado en el año 2014 con un ROE de 85,63\% ${ }^{8}$ el indicador de este grupo se deteriora a lo largo del tiempo de modo que al año 2018 llega a $-14,42 \%$ en 2018. Por su parte, las universidades estatales muestran un ROE intermedio, aunque relativamente bajo siendo de 3,30\% en el año 2014 y disminuyendo a 0,65\% en 2018.

Mediante la figura 3 se muestra la evolución del ROE según tipo de universidad.

8 En este grupo para el año 2014 se repite el efecto producido por los ROE fuera de rango de las Universidades Bolivariana $(1.945,27 \%)$ y La República $(442,33 \%)$. Si se extraen de la muestra ambas instituciones el ROE del grupo de universidades privadas-privadas para el año 2014 llegaría a 3,54\%. 


\section{Figura 3}

Evolución del ROE según tipo de universidad.

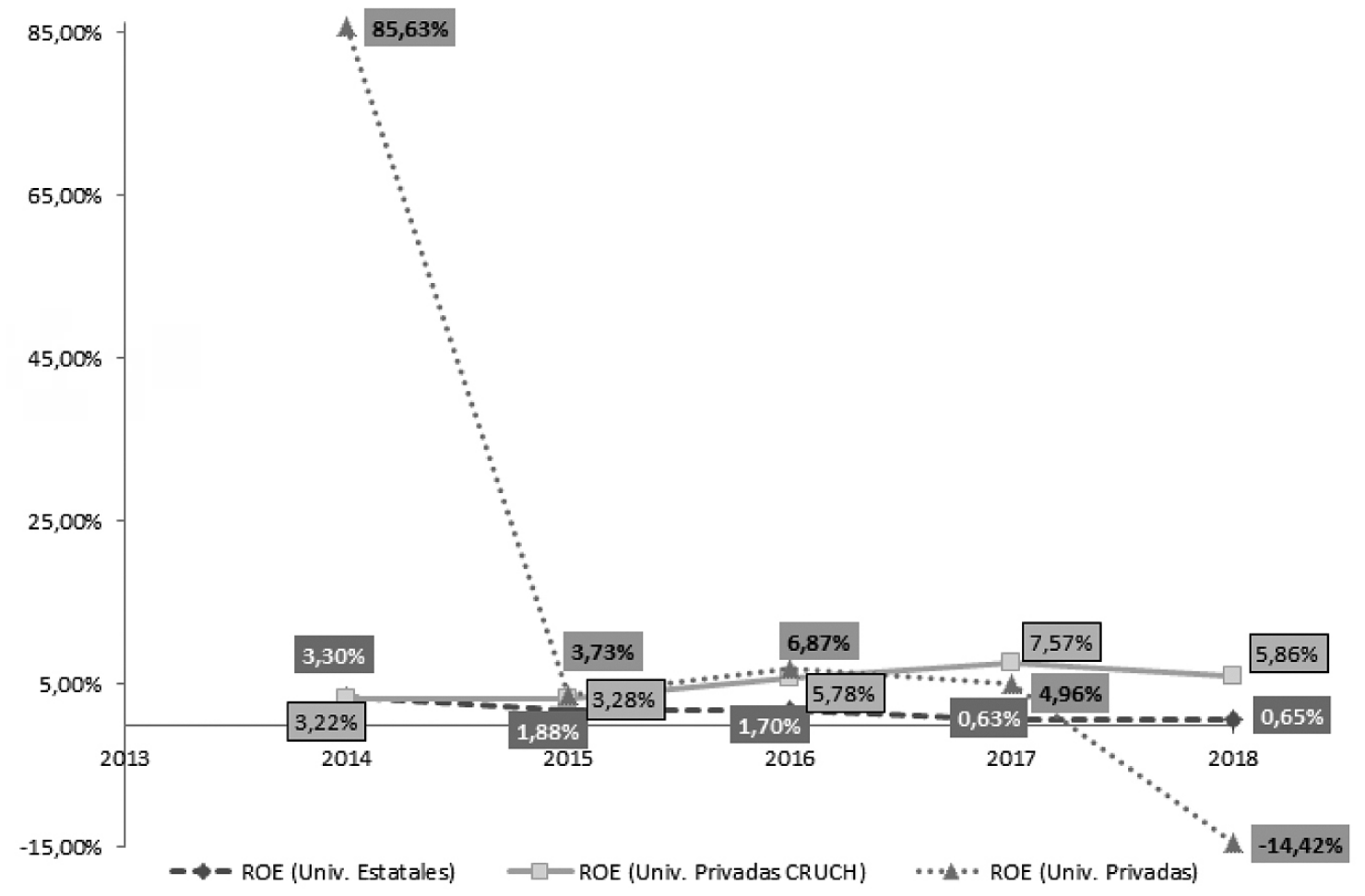

Fuente: Elaboración propia.

Revisando los indicadores componentes del ROE, en primer lugar, se aprecia el ratio de rentabilidad operacional. El grupo de universidades que evidencia el mejor desempeño a nivel operacional son las instituciones privadas pertenecientes al CRUCH. Este grupo muestra una rentabilidad operacional de 6,07\% al año 2014 con una leve tendencia al alza en los años posteriores alcanzando un 7,05\% al año 2018. Por su parte el grupo de universidades estatales muestra una relación EBIT/Ventas con tendencia a la baja siendo que al año 2014 este grupo de universidades mostraba un ratio de eficiencia operacional de 5,91\% para pasar a 1,21\% en el año 2018. Finalmente, el grupo de universidades privadas evidencia un recorrido más errático, considerando un ratio EBIT / Ventas de 2,54 al año 2014, pasando a ser negativo los años 2015 y 2016, volviendo a registros positivos con un 5,37\% y 3,59\% para los años 2017 y 2018 respectivamente. Mediante la figura 4 a continuación se presenta la evolución de la rentabilidad operacional por grupo de universidades. 


\section{Figura 4}

Evolución de la rentabilidad operacional según tipo de universidad.

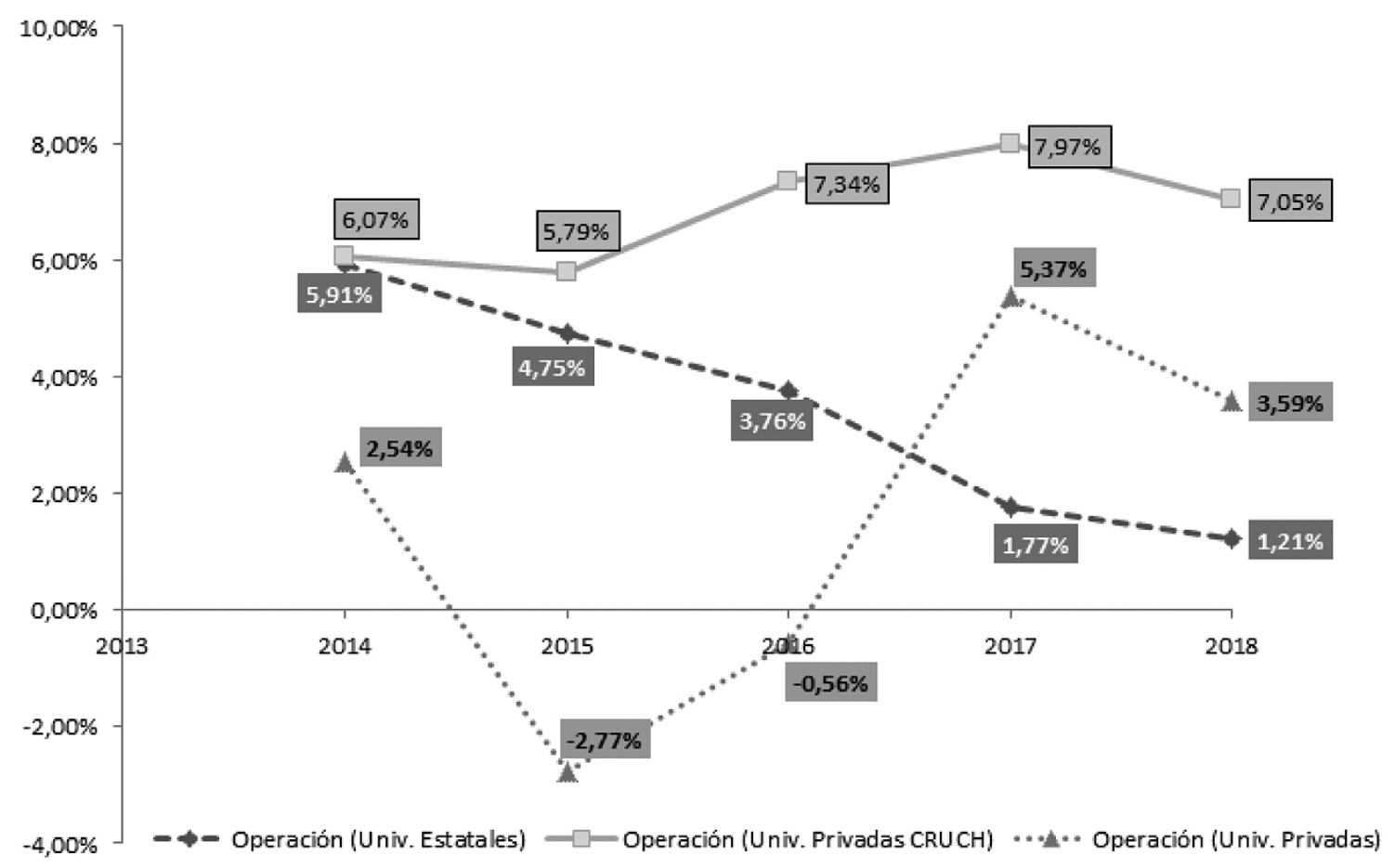

Fuente: Elaboración propia.

En relación a los indicadores de eficiencia medidos a partir de la rotación de activos, éstos son estables a lo largo del periodo para los tres grupos de universidades. El grupo de instituciones privadas es el que muestra el mejor desempeño de este indicador con un registro de 0,74 veces al año 2014 y de 0,81 veces para el año 2018. En segundo lugar, se encuentran las universidades privadas pertenecientes al CRUCH, grupo que al año 2014 registra un ratio de eficiencia de 0,56 veces mientras que al año 2018 anota 0,55 veces. Por último, es el grupo de universidades estatales el que muestra el peor desempeño en la rotación de activos dado que al año 2014 el ratio llega a 0,45 veces y para el año 2018 alcanza 0,46 veces. Mediante la figura 5 se representa la evolución del ratio de eficiencia por grupo de universidades. 


\section{Figura 5}

Evolución de la rotación de activos según tipo de universidad.

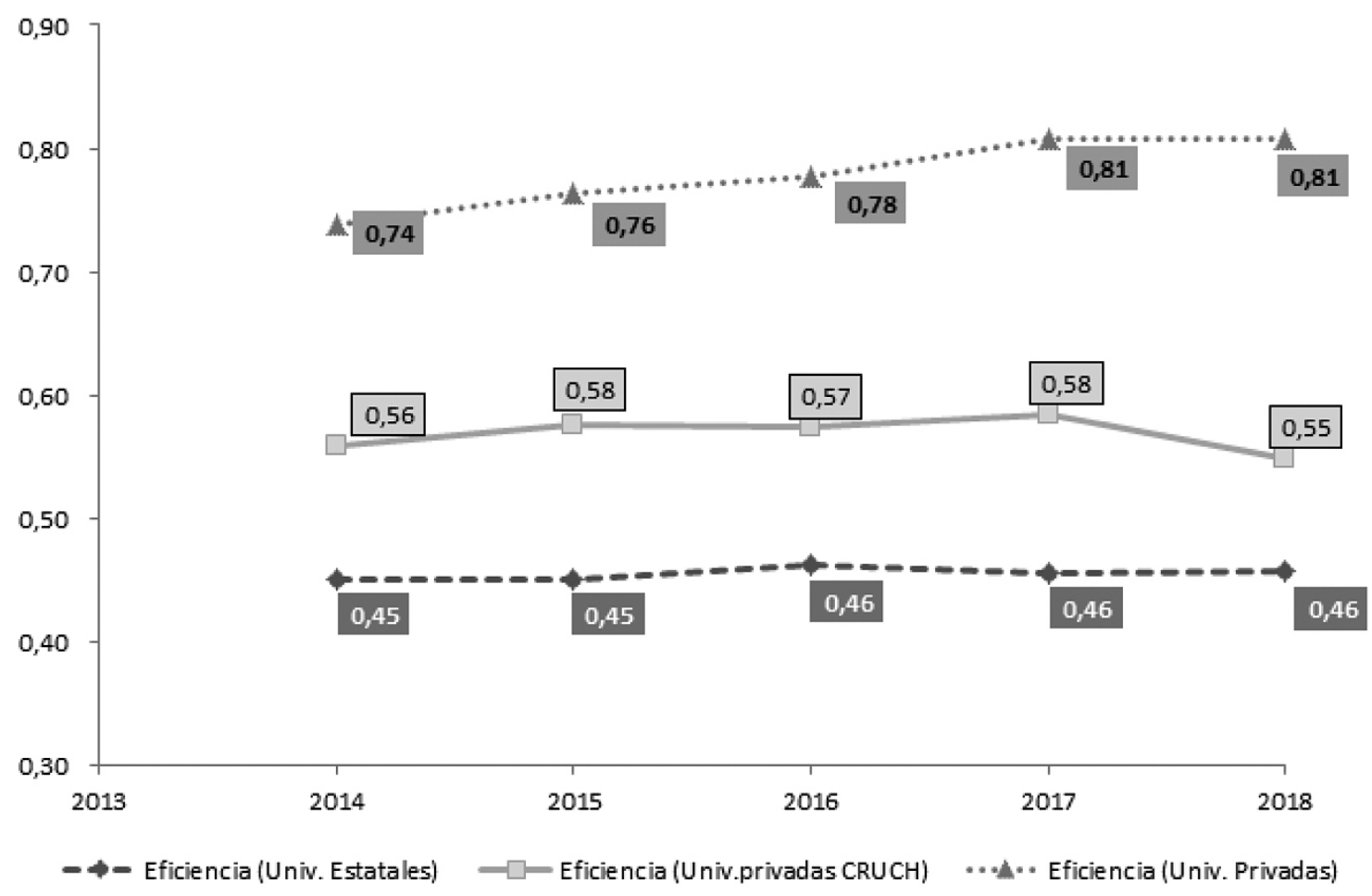

Fuente: Elaboración propia.

En el caso de la carga por intereses, el análisis revela que el grupo de universidades estatales es el que muestra los mejores resultados considerando que al año 2014 el indicador llega a 0,99 veces con tendencia al alza llegando a 1,52 veces en el año 2018. Los resultados de este grupo revelan la importancia que tiene para algunas instituciones estatales los resultados de carácter no operacional ya que el indicador registrará valores mayores a 1 siempre que EBT supere al EBIT. En estos casos las ganancias procedentes de fuentes no operacionales compensan de manera importante los costos financieros y por lo tanto el indicador arroja valores superiores a 1 . Sin perjuicio de lo anterior, resulta importante destacar lo que se podría considerar una falla del ratio de carga por intereses la cual se detecta en los casos en que las entidades han registrado pérdidas tanto a nivel operacional como antes de impuestos. Dado que la métrica de carga por intereses se calcula a partir de la relación EBT/EBIT, en los casos en que ambas líneas de resultado muestren pérdidas se obtendría un valor positivo del indicador incluso llegando a contribuir positivamente a la determinación del ROE. Ejemplo de esta situación queda en evidencia en el año 2018, donde la Universidad Arturo Prat (estatal) muestra EBT por -4.285 millones y EBIT por -2.677 , caso en que se obtiene un indicador de carga de intereses de 1,6 veces. En segundo lugar, se encuentran las universidades privadas pertenecientes al CRUCH, grupo que al año 2014 muestra un ratio de carga de intereses de 0,55 veces llegando a $-3,52$ veces al año 2018 . Vale mencionar que en este grupo el resultado del año 2018 se ve fuertemente explicado por los resultados de una sola insti- 
tución siendo el caso de la Universidad Técnica Federico Santa María. En el ejercicio 2018 dicha institución anotó un discreto resultado operacional de 33,9 millones de pesos frente a una pérdida antes de impuestos de -1.292 millones de pesos, resultados que explican el ratio de carga de intereses por $-38,12$ veces. Si se considera el resultado de esta entidad como outliers y se extrae de la muestra, la carga de intereses promedio de este grupo cambia a 0,8 siendo este resultado más cercano al de años anteriores. En tercer lugar, se encuentra el grupo de universidades privadas sin financiamiento estatal las que al año 2014 muestran un indicador de $-23,58$ veces y de 0,98 veces al año 2018. El ratio promedio para el año 2014 resulta atípico y se explica por los valores fuera de rango registrado por la Universidad Internacional SEK, entidad que en dicho ejercicio muestra un resultado operacional de 304 mil pesos y pérdida antes de impuestos de -209,6 millones de pesos, cifras que determinan una carga de intereses de $-689,41$ veces. Tal como en los casos anteriores, si se extrae de la muestra este outliers el ratio promedio de este grupo de entidades llega a 0,2 veces. Mediante la figura 6 a continuación se muestra la evolución del indicador carga de intereses por grupo de universidades.

\section{Figura 6}

Evolución de la carga por intereses según tipo de universidad.

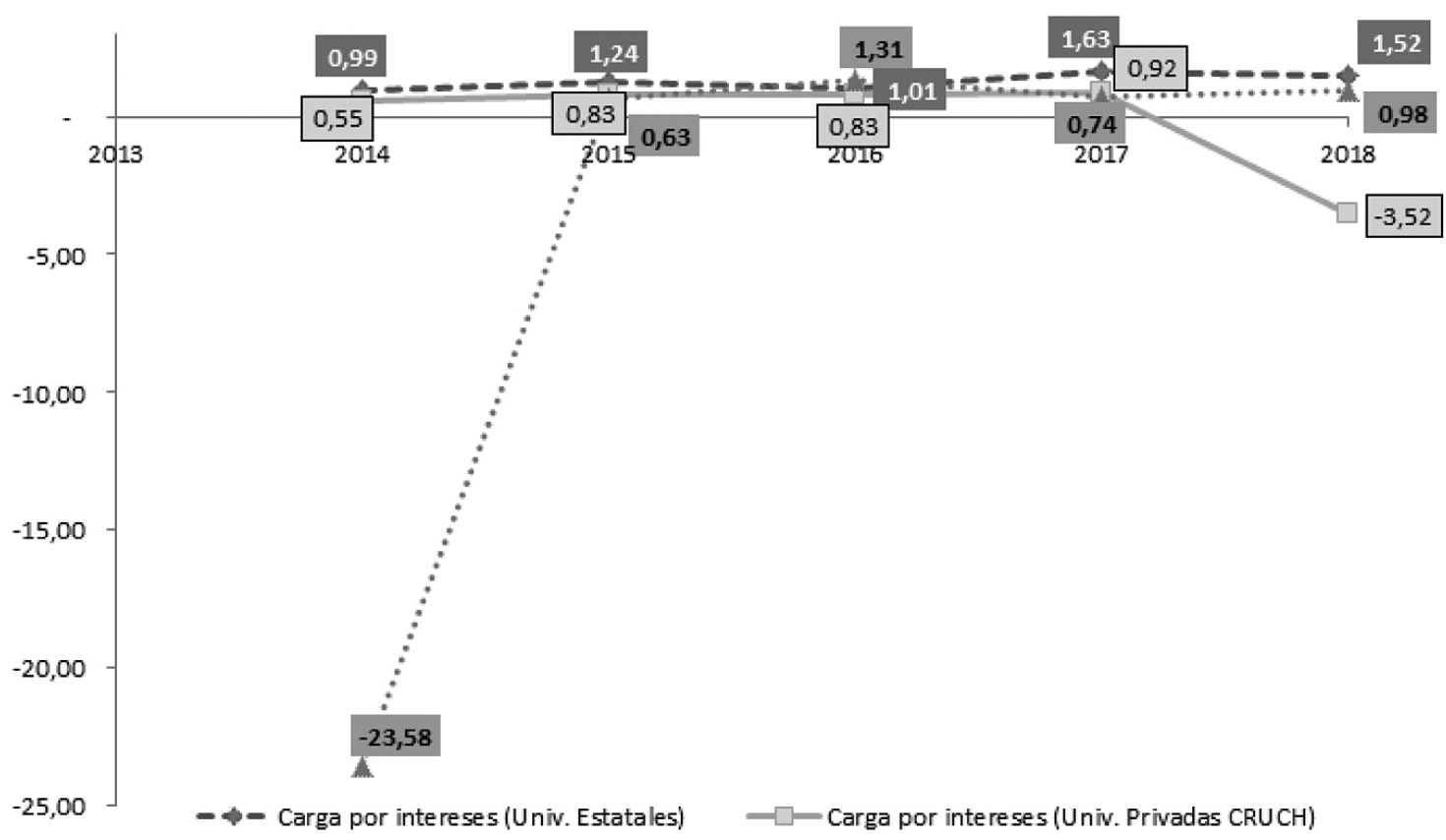

Fuente: Elaboración propia.

Finalmente, respecto al indicador de endeudamiento o solvencia se aprecia un menor apalancamiento financiero para los grupos de universidades estatales y privadas CRUCH. En el primer grupo se registra un indicador de solvencia estable a lo largo del tiempo considerando un ratio de 1,28 veces para el año 2014 y de 1,37 veces para el año 2018. En el caso de las entidades privadas CRUCH al año 2014 se muestra un indicador de solvencia de 1,71 veces y llegado a 1,64 veces al 
2018. Las entidades privadas evidencian mayores niveles de endeudamiento en consideración de los ratios de solvencia de 9,14 y 2,99 veces para los años 2014 y 2018 respectivamente. Mediante la figura 7 se muestra la evolución del indicador de solvencia por grupo de universidades.

\section{Figura 7}

Evolución del indicador de endeudamiento/solvencia según tipo de universidad.

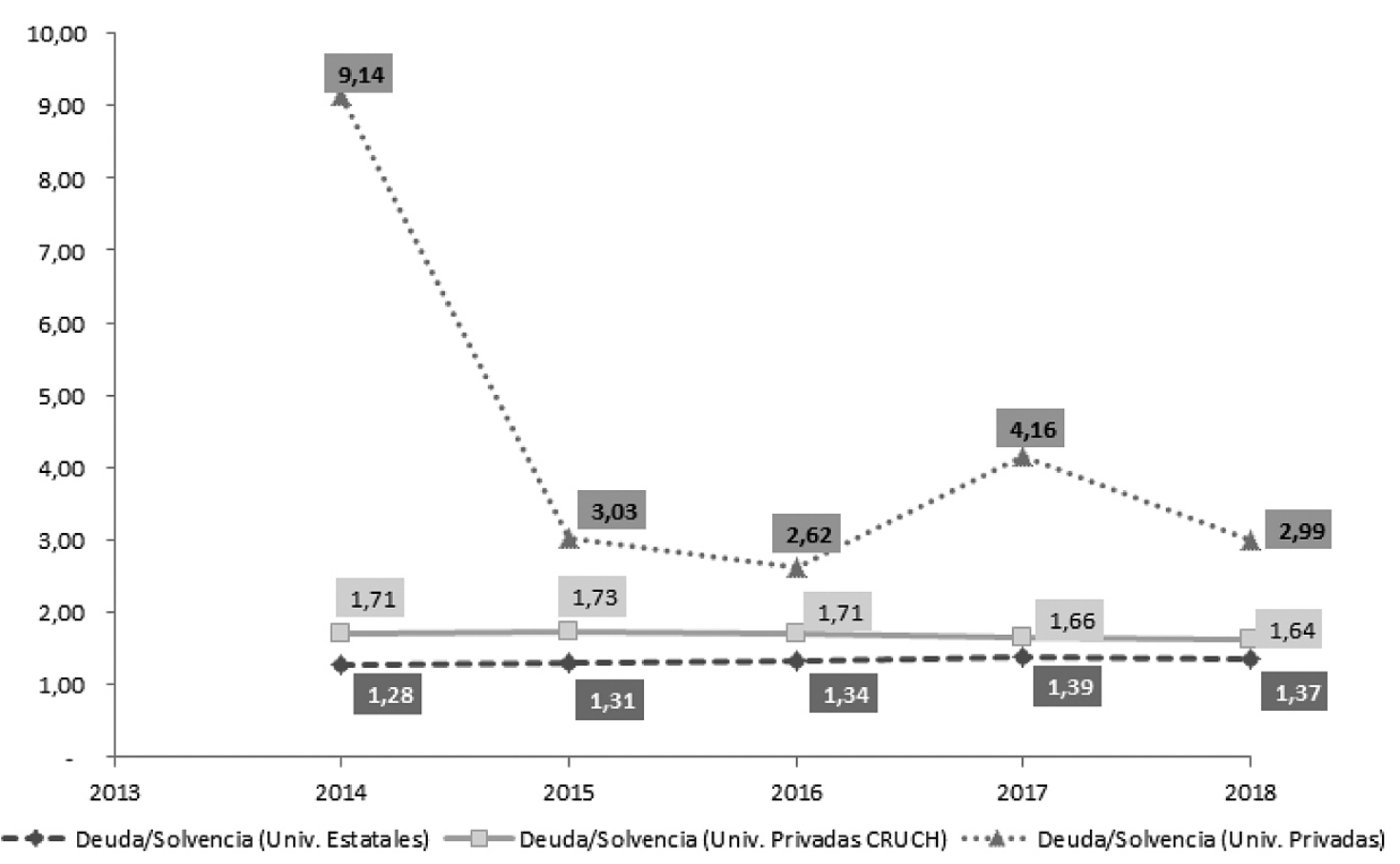

Fuente: Elaboración propia.

No se realizó un mayor análisis para el caso de los indicadores de carga tributaria ya que en general el resultado de este ratio es igual a 1 como promedio para todos los grupos de universidades y virtualmente nula dispersión a partir de los resultados de desviación estándar para cada grupo. Estos resultados confirman que los resultados netos de las universidades, en general, no se ven afectados por gastos de impuestos a la renta, aun cuando podrían generar ingresos que constituyen renta producto del desarrollo de actividades distintas a la educación.

\section{CONCLUSIÓN Y DISCUSIÓN DE RESULTADOS}

Se pudo observar que entre los años 2014 al 2018 las universidades chilenas han disminuido su rentabilidad a partir de la evolución del ROE y de esta métrica los indicadores relevantes en su desagregación son una baja rentabilidad operacional y baja rotación de activos. En promedio, las universidades se encuentran financiadas en un $43 \%$ por patrimonio y $57 \%$ por pasivos. Según tipo de universidades la que muestran un mayor ROE a lo largo del periodo son las universida- 
des privadas $\mathrm{CRUCH}$, seguidas por los planteles estatales y finalmente las entidades totalmente privadas. Desagregando el ROE según los indicadores de eficiencia interna revisados, son las universidades privadas CRUCH las que anotan una mayor rentabilidad operacional, seguida por las entidades privadas-privadas. Las instituciones estatales son las que muestran el peor desempeño operacional en el periodo. Observando el ratio de carga por intereses financieros son las universidades estatales las que muestran el mejor resultado, seguido por las universidades privadas-privadas, y en último término se encuentran a las universidades privadas CRUCH. En cuanto a la métrica de solvencia, son las entidades privadas-privadas las que muestran mayores niveles de apalancamiento, seguidas por las universidades privadas $\mathrm{CRUCH}$ y en tercer lugar se encuentran los planteles estatales.

Luego de una amplia revisión de la información financiera de las universidades chilenas, se observan oportunidades de ampliar las líneas de investigación aplicadas al sector. La información disponible permite proyectar estudios relacionados por ejemplo con la calidad de la información contenida en los estados financieros, o bien el desarrollo de modelos predictivos o de alerta temprana en cuanto a los niveles de solvencia de las entidades.

\section{REFERENCIAS BIBLIOGRÁFICAS}

Berstein L \& Wild J. (1998). Financial Statement Analysis: Theory, Application, and Interpretation. Boston: McGraw-Hill.

CNA-Chile. (2014). Sustentabilidad Financiera: Información relevante y proceso. Santiago.

CNA-Chile. (2018, 06 26). www.cnachile.cl. Retrieved 01 13, 2020, from https: / www.cnachile.cl/ noticias / Paginas / SUSTENTABILIDAD-FINANCIERA.aspx

Dehning \& Stratopoulos. (2002). DuPont analysis of an IT- enabled competitive advantage. International Journal of Accounting Information Systems.

Gitman L. (2003). Principios de Administración Financiera. México.

Gutiérrez Hidalgo F. (2005). Evolución Histórica de la Contabilidad de Costos y de Gestión. Spanish Journal of Accounting History.

OCDE. (2004). On Edge: Securing a Sustainable Future for Higher Education. Paris: OECD Publications.

Revsine L., Collins D. \& Johnson W. (2005). Financial Reporting and Analysis. New York: Pearson/ Prentice Hall.

Stickney C.\& Brown P. (1999). Financial Reporting and Statement Analysis: A Strategic Perspective. New York: Dryden Press.

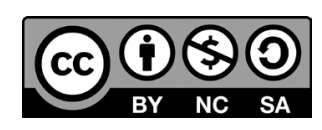

Esta obra está bajo una licencia de

Creative Commons Atribución-NoComercial-CompartirIgual 4.0 Internacional 